\title{
Impact and Risk Analysis of Wind Farm on Environment
}

\author{
Wang Haiwen \\ Powerchina Guiyang Engineering Corporation Limited, Guizhou, Guiyang 550081
}

Keywords: ecological damage; noise; safety of birds; risk; wind farm

\begin{abstract}
With the shortage of energy supply and increasingly serious environmental problems, wind power industry ushered in rapid development. The operating noise of the fan is low frequency noise, which may cause some diseases to human. At the same time, wind farms have potential effects on electromagnetic radiation and regional visual landscape. However, under normal circumstances, except for a certain risk of collision for a small number of birds, the risk is very low, which will not cause irreversible risks to the environment, human health or animals, etc.
\end{abstract}

\section{Introduction}

Wind power is widely used and developing rapidly, mainly because it is a clean renewable energy source, which to some extent reduces emissions of harmful gases, relieves pressure on energy supply and slows the greenhouse effect. However, the rapid development of wind power has a potential impact on birds, regional ecological vegetation and species diversity ${ }^{[1]}$.

This paper would briefly introduce the situation of wind power in China, analyze its impact on ecological environment, noise, bird safety, electromagnetic radiation and landscape combining with related research and investigation at home and abroad, and further discuss the risks of various impacts.

\section{Analysis of the impact of wind farms on the environment}

\subsection{Ecological damage}

The destruction of wind farms on ecology is mainly reflected in the construction period, in which the disturbance and massive destruction of surrounding land surface and vegetation will cause a lot of soil erosion ${ }^{[2]}$. During construction preparation, site formation and equipment stacking would change or destroy the soil structure and reduce the soil erosion resistance of the landform, which will easily lead to soil erosion. Maintenance and construction during the process would cause large area and volume disturbance to the road, destroying the large area of surface soil structure.

\subsection{Noise impact}

The noise in the fan operation mainly includes mechanical noise and aerodynamic noise. The mechanical noise is mainly generated by the electricgenerator and gear box.Aerodynamic noise is mainly the interaction between the turbine blade and surrounding air. The noise of gearbox is caused by the vibration and friction of the meshing gears. For the same acoustic point around the fan, the noise value will increase with the increase of wind speed and decrease with the increase of distance [3]. When the fan is running, its noise presents obvious low-frequency characteristics, and with the increase of the output power of the wind turbine, the noise generated also increases and changes to a lower frequency. Meanwhile, as the rotation speed of the unit increases, the noise of the fan will increase gradually, and a good linear relationship exists.

\subsection{Bird impact}

During the operation of the wind farm, the birds will be affected, the most direct is the occurrence of bird collision. The location of the wind farm has something to do with the probability of a bird collision. When the wind farm is located or near the migration channel of birds, the probability of collision will be greater. The probability of a collision increases when the wind farm 
is close to an area where birds are concentrated. In the monitoring study of Horns Rev wind farm in Denmark, it was found that the distribution of birds within $2 \sim 8 \mathrm{~km}$ around the wind farm changed, and the occurrence frequency of birds in the region decreased significantly [4].

\subsection{Electromagnetic radiation and visual landscape}

The electromagnetic pollution of wind power plants comes from substations and transmission lines, which will cause the signal-to-noise ratio of electronic equipment, communication facilities, etc. to be greatly reduced or even unable to work. At the same time, the background noise of the electromagnetic environment in the wind farm area may be elevated by the generated electromagnetic radiation, and have occlusion and reflection effect on the electromagnetic signal, which can lead to the effect of frequency system efficiency in key regions or regions, and even affect the economic construction and national defense construction of the whole region and even the country.

\section{Risk analysis}

\subsection{Ecology and noise}

The impact of wind farm on ecology is mainly reflected in the construction period. Due to the wind turbine, base tower and road construction during the project construction, the original topography of the area was damaged greatly, and the soil structure was changed, resulting in temporary soil erosion and soil fertility decline.

However, in the construction process, the application of relevant measures and the timely ecological restoration measures after completion can minimize the impact of ecological environment and reduce risks

The research result of wind farms in Guhuitengxile area of Inner Mongolia shows that wind farm construction has the certain impact on regional plant community species, but the impact is short-term and temporary impact and will not cause permanent or irreversible ecological damage. We did a long-term observation and research on wind farms built in 1996, 1999, 2002, 2006 and 2009 were carried out in the study. Through the observation of changes in the number of wind farm species built for different years, although the construction has affected vegetation and regional species, the number of species has been reduced and changed. [5] However, as time goes on, the number of species will gradually increase, and a small number of new species will be produced. At the same time, soil structure and vegetation community structure will gradually recover and improve. In general, after a certain period of recovery, although it will cause a certain degree of small changes and differences compared with the original appearance, for the regional ecology as a whole, the functional of the ecological environment and the coverage of regional vegetation can be compared. Comprehensive recovery.

\subsection{Bird safety aspects}

The most direct and serious impact of the presence of wind farms on birds is the collision of birds and wind turbines leading to injuries and deaths. Studies indicate that if the construction area of the wind farm is on the flight path of birds, there is a risk of collision and death of the wind turbine during flight and migration. The incidence of this risk depends on the flight height of the birds.

During the migration flight, birds can change their flight direction to avoid flying and feeding. Some birds even adapt to the habitat after the wind farm is built. Scientists in Ireland have studied the effects of wind turbines on bird collisions and migration. The long-term observation of bird migration through the radar to the Horns Rev electric field in the world's largest wind farm shows that birds have the ability to avoid the certain wind farms.For example, when a flock of northbound birds is about 400meters away from the wind farm, the birds begin to change their flight direction and head west.Researchers such as Bech and Petersen looked at bird migration in the vicinity of wind farms. During the migration season, most birds fly around wind farms on their own to avoid 
the area.

\subsection{Electromagnetic radiation and visual landscape}

Electromagnetic radiation generated by the construction and operation of wind farms will cause excessive intensity of electromagnetic field in local space or in the whole space, which will have certain impact on some equipment, but will not cause pollution risk.

With the rapid development of wind power industry, more and more attention has been paid to the environmental design concept in the construction of wind power plants. Based on the integration of wind power plants into the surrounding environment, the wind power plants have gradually developed into a virtuous circle that further adds interest to the natural rural landscape, thus adding vitality to the region. As early as in 2003, wind power installations were allowed in parks in Japan. In the aspect of landscape, the landscape design was successfully integrated into the appearance design of wind farm. On the basis of satisfying the sustainable power generation without polluting the environment, the overall appearance design of the wind farm is more ornamental. At the same time, as a member of the wind farm design team, the landscape architect in the UK designs the visual effects of the wind farm from different angles, highlighting the integration with the surrounding environment.

\section{Discussion}

From the analysis of potential impacts of wind farms on regional ecological environment, noise, bird safety, electromagnetic radiation and visual landscape, it is not difficult to see that the construction period and operation period of wind farms have certain impacts on these aspects to different degrees. Although construction and operation of wind farm will have impact to nature environment in a certain degree, human health and the birds. However, if landscape design can be integrated into wind farm appearance design, optimize site selection and layout reasonably in the design stage, effective ecological protection, impact mitigation and related control measures will be taken in the construction process, and relevant engineering measures and plant measures will be taken in key areas, which can minimize the impact during the construction. After the completion of the construction, timely restoration of vegetation restoration measures and plant diversity restoration measures can minimize the occurrence probability of the impact and even avoid it.

However, most of the construction workers in China's current stage have not undergone formal professional training, low level of education and technologyand lack of relevant professional knowledge and awareness. To a different extent, there are non-compliance with discipline, not completely in accordance with the processor even illegal operations. At the same time, the enterprises and related units have insufficient education on the safety management awareness of construction workers, the implementation of the responsibility system is not in placeand the imperfections of related management and other reasons have also led to various dirty, chaotic and poor construction sites. The problem arises, which in turn increases the risk value of various adverse effects. In general, if all aspects of wind farm construction can effectively control and implement various relevant measures, the potential impacts of wind farms are non-existent or the risk value will be very low.

\section{Conclusion}

The use of wind power will have a certain potential impact on regional ecological environment, birds and human health while alleviating the energy crisis and reducing environmental pollution. However, a large number of studies and surveys show that there is a certain risk of collision for only a small number of birds. In other cases, the probability of adverse effects is very low under normal circumstances. 


\section{References}

[1] Global Wind Energy Council (2016), 2016 Global Wind Energy Development Outlook Report

[2] Council G W E.2013. Global wind statistics 2012 report. Brussels, Belgium: Global Wind Energy Council, 1-4

[3] China Wind Energy Association (CWEA) (2015), 2014 China Wind Power Installed Capacity Statistics, Wind Energy, 6(2): 36-49

[4] Yanfen Zhou, Yujie Geng et al. (2011), Environmental Impacts and Control of Wind Farms, Hubei Agricultural Sciences, 50(13)

[5] Huaifeng Cui, Qian Yang, et al. (2008), Analysis of factors affecting the collision of birds and wind turbines and their protective measures, Environmental Science Guide, 27(4), P52-56 